\title{
Meeting report: Frontiers in genetics: genomics and epigenomics
}

\author{
Kyudong Han $\cdot$ Chul Geun Kim $\cdot$ Nam-Soo Kim
}

Received: 22 July 2013/Accepted: 24 July 2013/Published online: 8 August 2013

(C) The Genetics Society of Korea 2013

The second meeting of the Genetics Society of Korea's mini symposium series was held at Hanyang University in Seoul on May 24, 2013. Thanks to the advanced technology for DNA sequencing, the field of genetics and genomics has been rapidly developed and highlighted. The goal of the symposium was to understand technology which could be used in studying genetics and genomics, and share the recent findings that we have unveiled by the large genome data. Six prominent researchers presented their studies at the symposium series titled "Frontiers in Genetics: Genomics and Epigenomics" and the presented topics were very diverse and remarkable.

The first speaker, Dr. Kyudong Han, Department of Nanobiomedical Science, Dankook University, Korea and DKU-Theragen Institute for NGS analysis, Korea, discussed the impact of mobile genetic elements on the genomic rearrangements, focusing on the elements as a major source causing genomic difference between different species and even within a species. Unveiling the role of transposable elements in genome evolution would be one of the significant achievements of the many remarkable findings from the genomics analyses. Many studies

K. Han

Department of Nanobiomedical Science \& WCU Research

Center, Dankook University, Cheonan 330-714, South Korea

C. G. Kim

Department of Life Science, Hanyang University,

Seoul 133-791, South Korea

\section{N.-S. Kim ( $\square)$}

Department of Molecular Bioscience, College of Biomedical Science, Institute of Bioscience and Biotechnology,

Kangwon National University, Chuncheon 200-701,

South Korea

e-mail: kimnamsu@kangwon.ac.kr including human whole genome sequencing projects (CSAC 2005; Gibbs et al. 2007; Lander et al. 2001), 1000 genome project (Abecasis et al. 2010), and ENCODE project (Dunham et al. 2012) have consistently shown that mobile genetic elements account for $\sim 45 \%$ of the human genome. The mobile genetic elements are largely divided into two classes; DNA transposons and retrotransposons (Deininger and Batzer 2002; Smit 1996). Currently, various primate reference genome sequences (human, chimpanzee, and rhesus macaque) are available for comparative genomics. The combined method of computational data mining and experimental verification allows us to identify and characterize species-specific mobile genetic elements in the primate genomes (Han et al. 2007a, b, 2008; Lee et al. 2007; Sen et al. 2006). Through those studies, he presented evidences that mobile genetic elements are still active to retrotranspose in their host genomes and thus have a potential to cause genomic variations and rearrangements (Brouha et al. 2003; Han et al. 2008; Sen et al. 2006). In reality, it has been reported that those genomic alterations caused by mobile genetic elements have increased the genomic diversity between human and non-human primates. In addition, the mechanisms which mobile genetic elements use to modify human and non-human primate genome have been well studied and the mechanisms include de novo mobile genetic element insertion, mobile genetic element insertion-mediated deletion, and the homologous recombination between the elements.

In the symposium, Dr. Han reported that L1 recombination-associated deletion (L1RADs) is one of factors generating human genomic variation (Han et al. 2008). L1 (Long INterspersed Element-1 or LINE-1) is the most ubiquitous non-LTR retrotransposon in eukaryotic genomes, accounting for $\sim 17 \%$ of the human genome (Lander et al. 2001). Dr Han and his colleagues identified 
73 human-specific L1RAD events. Since the divergence of the human and chimpanzee lineages $(\sim 6$ million years ago), the L1RAD events have deleted $\sim 450 \mathrm{~kb}$ of the human genome via two different deletion mechanisms (55 nonallelic homologous recombination and 18 nonhomologous end joining between two L1s) (Han et al. 2008). Based on his findings, Dr. Han alleged that mobile genetic elements have a high impact on modifying primate genomes and thus play a significant role in the dynamics of the human and non-human primate genomes.

The second speaker, Dr. Heui-Soo Kim (Department of Biological Sciences, Pusan National University) introduced their recent findings on the human endogenous retroviruses (HERVs). HERV is dispersed throughout the mammalian genomes with various forms: full-length HERV, solitaryLTR, and truncated HERV. In the symposium, he presented the function of long terminal repeats (LTRs) of HERVs. LTRs contain transcriptional regulatory elements, promoters, enhancer, and polyadenylation signals, which could affect the transcription of specific cellular genes (Kim 2012). In addition, the HERVs also have critical roles in diversification, speciation, and evolution. Dr. Kim suggested various evidences that HERV could alter the structure and expression of cellular genes in the human and non-human primate genomes. In his presentation, he stated that mobile genetic elements could cause alternative splicing which lead to diverse transcripts of a gene in their host genomes. In reality, he found that a mobile genetic element, MaLR, was integrated into the exon10 of $P C D H 11 X / Y$ during the primate evolution and caused alternative splicing of the gene. $P C D H 11 X$ and $P C D H 11 Y$ have eight and four transcripts, respectively, and their alternative transcripts have been expressed differently in different human tissues (Ahn et al. 2010). Retroelements integrated in the common ancestor of human and mouse have created various periphilin transcripts in the two species; human and mouse have ten and two periphilin transcripts, respectively. Periphilin gene locates on chromosome 12q12 and one of HERV-M elements exists within the gene in a truncated form composed of $5^{\prime}$ LTR-gag-pol-3'LTR. This HERV-M affects the transcription of periphilin gene leading to its transcript variants $1-3$ in the human genome (Huh et al. 2006).

It was recently suggested that alternative transcripts could be used as a biomarker for cancers because mobile genetic elements are one of the factors altering the expression pattern of cellular genes. DYXICl gene has three alternatively spliced transcripts and one of the transcripts result from HERV-H LTR existing within the last exon of the respective transcript. The expression pattern of the transcripts is different between normal and cancer tissues. Thus, it was suggested that the alternatively spliced transcript variants of $D Y X 1 C l$ gene could be used as a cancer biomarker (Kim et al. 2009). In addition, the Choroideremia $(C H M)$ gene has two transcript variants, $C H M$ isoform a and b. LTR12C element existing in the gene confer an alternative splicing site on the gene which result in $C H M$ isoform b. It was reported that $C H M$ isoform $\mathrm{b}$ is highly expressed in colon tumor tissues compared with its normal tissues (Jung et al. 2011). Thus, the high expression of the CHM isoform b could be used as a marker for a colon cancer.

The third speaker, Dr. Jong Bhak (Theragen Bio Institute and Genome Research Foundation) gave a presentation entitled 'Close Species Genomics'. In the beginning of his talk, he thanked researchers for their honesty and passion in performing science, tax payers, TBI \& Genome Research Foundation, and Genetics Society of Korea for supporting scientific research. Dr. Bhak said, "Useful to do close but distinct species genome comparison to detect geno-phenotype associations easily" and "More sequencing for species around references". He emphasized the importance of comparative genomics and bioinformatics for understanding a precise genomic mapping and structure. Since the ostentatious achievement of human genome project, more human individual genomes have been sequenced. Next generation sequencing (NGS) technique has accelerated whole genome sequencing, RNA sequencing, and Exome sequencing. The advanced sequencing technology challenges personal genome sequencing for clinical application.

Dr. Bhak's presentation was ended up with 'Tiger genome project'. To investigate geno-phenotype association between tiger and its close relatives belonging to the genus Panthera, the de novo assembled whole genome sequence of an Amur tiger (Panthera tigris altaica) was constructed using Illumia HiSeq2000 platform. Currently the genome sequences of white tiger, African lion (Panthera leo), white African lion, and snow leopard (Panthera uncia) are available for comparative genomics. Through the comparison of the domestic cat and other big cat reference genome sequences, they found molecular adaptation which is consistent with the big cats' adaptation. The big and domestic cat genomes are very similar which reflects the recent radiation of the 37 modern cat species $(<11$ million years ago). The composition of repeat elements is extremely similar between the domestic and big cat genomes. The high degree of genomic synteny between the two genomes indicates the strong genomic conservation between the two cats.

The fourth speaker, Dr. Jung-Shin Lee (Department of Molecular Bioscience, Kangwon National University) talked about 'Context dependent histone modification during transcription elongation'. Monoubiquitination of histone H2B on Lys 123 (H2BK123ub) is a transient histone modification considered to be essential for 
establishing histone $\mathrm{H} 3$ lysine 4 (H3K4) and H3K79 trimethylation by Set1/COMPASS and Dot1, respectively. COMPASS, the yeast homologue of the mammalian MLL complex, is a H3K4 methylase consisting of Set 1 and seven other polypeptides named Cps60-Cps15. She demonstrated that histone $\mathrm{H} 2 \mathrm{~B}$ monoubiquitination controls the association of the only essential subunit of COMPASS, Cps35, within the complex. Cps35 is required for COMPASS' catalytic activity in vivo and the addition of exogenous purified Cps35 to COMPASS purified from a rad6 background results in the generation of a methylation competent COMPASS. Cps35 associates on the chromatin of COMPASS-regulated genes in a H2BK123 monoubiquitinationdependent, Set1-independent manner. These findings offer insight into the molecular role of Cps35 in translating the H2B monoubiquitination signal into H3 methylation. In addition, she identified Chd 1 as a factor that is required for the maintenance of high levels of H2B monoubiquitination, but not for H3K4 and H3K79 trimethylation. Loss of Chd1 results in a substantial loss of H2BK123ub levels with little to no effect on the genome-wide pattern of H3K4 and H3K79 trimethylation. She also suggested that the function of Chd1 in maintaining H2BK123ub levels is conserved from yeast to humans. Her study provides evidence that only small levels of H2BK123ub are essential for full levels of $\mathrm{H} 3 \mathrm{~K} 4$ and $\mathrm{H} 3 \mathrm{~K} 79$ trimethylation in vivo and points to a possible role for Chd1 in positively regulating gene expression through promoting nucleosome reassembly coupled with H2B monoubiquitination.

The fifth speaker, Dr. Jae-Bum Bae (Division of Structural and Functional Genomics, Epigenomics Team, Korea National Institute of Health) gave a presentation about 'Epigenomic difference on disease-discordant monozygotic twins' focusing on the epigenomic studies about monozygotic twins, discordant for rheumatoid arthritis (RA) and diabetes (T2D). He emphasized that the genome-wide epigenomic study is a powerful and successful tool to discover epigenetic changes associated with the phenotypic traits of discordant monozygotic twins. Using infinium $450 \mathrm{~K}$ high density methylation array and MBD-Seq (Methyl-CpG binding domain protein-Sequencing), his team examined DNA methylation patterns in the DNA samples of 743 monozygotic twins, and found $8 \mathrm{RA}$ and $12 \mathrm{~T} 2 \mathrm{D}$ discordant monozygotic twins. One of previous epigenomic studies identified ten putative differentially methylated positions (DMPs) associated with RA (Liu et al. 2013). Among them, five DMPs belong to C6orf10 gene (Liu et al. 2013). Dr. Bae's group has been trying to identify useful markers which could be used to detect RA. They examined the DNA methylation variation between the $\mathrm{CD} 4^{+} \mathrm{T}$ cells of a normal individual and an individual with RA, focusing on three markers which were identified in $\mathrm{RA} \mathrm{CD} 4^{+} \mathrm{T}$ cells, and found that two, Sox 2 and AMOTL1, out of the three markers are differentially methylated in monozygotic twins, discordant for RA. In addition, they conducted whole genome bisulfate sequencing to study monozygotic twins discordant for T2D and found 28 out of 62 T2D GWAS lociexhibiting significant DNA methylation difference ( $p$ value $<0.05$ ) in the monozygotic twins discordant for T2D. Dr. Bae stated that further analysis of the 28 candidate loci will identify epigenetic changes which could be the cause of T2D.

The sixth speaker, Dr. Ho Chul Kang (Department of Physiology, Ajou University School of Medicine and Department of Neurology and Institute for Cell Engineering, Johns Hopkins University School of Medicine) reported 'Iduna (RNF146), a novel PAR polymer-induced cell death (PATHANATOS) inhibitor'. He and his colleagues previously identified RNF146 as an $N$-methylD-aspartate (NMDA) glutamate-receptor inducible gene (Hong et al. 2004) and then renamed the RNF146 to Iduna which is neuroprotective against glutamate NMDA receptor-mediated excitotoxicity both in vitro and in vivo, and protects against parthanatos via binding to poly(ADPribose) (PAR) (Andrabi et al. 2011). The protective effects of Iduna are independent and downstream of PAR polymerase- 1 activity. A mutation at the PAR polymer binding site completely destroys the RNA binding activity of Iduna and attenuates its protective actions. Iduna is protective in vivo against NMDA-induced excitotoxicity and middle cerebral artery occlusion-induced stroke in mice (Andrabi et al. 2011). These results suggest that Iduna is the first known endogenous inhibitor of pathanatos. In addition, his group and their coworkers from Johns Hopkins University developed a 17,000 Human Proteome Chip on a single glass slide which covers $80 \%$ of the human proteome.

We thank the organizers, Chul Geun Kim and Kyudong Han, for bringing this science community together to network and discuss their research findings at the second meeting of Genetics Society of Korea's mini symposium series.

\section{References}

Abecasis GR, Altshuler D, Auton A, Brooks LD, Durbin RM, Gibbs RA, Hurles ME, McVean GA (2010) A map of human genome variation from population-scale sequencing. Nature 467:1061-1073

Ahn K, Huh JW, Kim DS, Ha HS, Kim YJ, Lee JR, Kim HS (2010) Quantitative analysis of alternative transcripts of human PCDH11X/Y genes. Am J Med Genet B 153B:736-744

Andrabi SA, Kang HC, Haince JF, Lee YI, Zhang J, Chi Z, West AB, Koehler RC, Poirier GG, Dawson TM, Dawson VL (2011) Iduna protects the brain from glutamate excitotoxicity and stroke by interfering with poly(ADP-ribose) polymer-induced cell death. Nat Med 17:692-699 
Brouha B, Schustak J, Badge RM, Lutz-Prigge S, Farley AH, Moran JV, Kazazian HH Jr (2003) Hot L1s account for the bulk of retrotransposition in the human population. Proc Natl Acad Sci USA 100:5280-5285

CSAC (2005) Initial sequence of the chimpanzee genome and comparison with the human genome. Nature 437:69-87

Deininger PL, Batzer MA (2002) Mammalian retroelements. Genome Res 12:1455-1465

Dunham I, Kundaje A, Aldred SF, Collins PJ, Davis CA, Doyle F, Epstein CB, Frietze S, Harrow J, Kaul R et al (2012) An integrated encyclopedia of DNA elements in the human genome. Nature 489:57-74

Gibbs RA, Rogers J, Katze MG, Bumgarner R, Weinstock GM, Mardis ER, Remington KA, Strausberg RL, Venter JC, Wilson RK et al (2007) Evolutionary and biomedical insights from the rhesus macaque genome. Science 316(5822):222-234

Han K, Konkel MK, Xing J, Wang H, Lee J, Meyer TJ, Huang CT, Sandifer E, Hebert K, Barnes EW et al (2007a) Mobile DNA in old world monkeys: a glimpse through the rhesus macaque genome. Science 316:238-240

Han K, Lee J, Meyer TJ, Wang J, Sen SK, Srikanta D, Liang P, Batzer MA (2007b) Alu recombination-mediated structural deletions in the chimpanzee genome. PLoS Genet 3:1939-1949

Han K, Lee J, Meyer TJ, Remedios P, Goodwin L, Batzer MA (2008) L1 recombination-associated deletions generate human genomic variation. Proc Natl Acad Sci USA 105:19366-19371

Hong SJ, Li H, Becker KG, Dawson VL, Dawson TM (2004) Identification and analysis of plasticity-induced late-response genes. Proc Natl Acad Sci USA 101:2145-2150

Huh JW, Kim TH, Yi JM, Park ES, Kim WY, Sin HS, Kim DS, Min DS, Kim SS, Kim CB et al (2006) Molecular evolution of the periphilin gene in relation to human endogenous retrovirus $m$ element. J Mol Evol 62:730-737

Jung YD, Huh JW, Kim DS, Kim YJ, Ahn K, Ha HS, Lee JR, Yi JM, Moon JW, Kim TO et al (2011) Quantitative analysis of transcript variants of CHM gene containing LTR12C element in humans. Gene 489:1-5

Kim HS (2012) Genomic impact, chromosomal distribution and transcriptional regulation of HERV elements. Mol Cells 33: 539-544

Kim YJ, Huh JW, Kim DS, Bae MI, Lee JR, Ha HS, Ahn K, Kim TO, Song GA, Kim HS (2009) Molecular characterization of the DYX1C1 gene and its application as a cancer biomarker. J Cancer Res Clin Oncol 135:265-270

Lander ES, Linton LM, Birren B, Nusbaum C, Zody MC, Baldwin J, Devon K, Dewar K, Doyle M, FitzHugh W et al (2001) Initial sequencing and analysis of the human genome. Nature 409: $860-921$

Lee J, Cordaux R, Han K, Wang J, Hedges DJ, Liang P, Batzer MA (2007) Different evolutionary fates of recently integrated human and chimpanzee LINE-1 retrotransposons. Gene 390:18-27

Liu Y, Aryee MJ, Padyukov L, Fallin MD, Hesselberg E, Runarsson A, Reinius L, Acevedo N, Taub M, Ronninger M et al (2013) Epigenome-wide association data implicate DNA methylation as an intermediary of genetic risk in rheumatoid arthritis. Nat Biotechnol 31:142-147

Sen SK, Han K, Wang J, Lee J, Wang H, Callinan PA, Dyer M, Cordaux R, Liang P, Batzer MA (2006) Human genomic deletions mediated by recombination between Alu elements. Am J Hum Genet 79:41-53

Smit AF (1996) The origin of interspersed repeats in the human genome. Curr Opin Genet Dev 6:743-748 